\title{
Multi-Detector X-Ray Mapping and Generation of Correction Factor Images for Problem Solving
}

\author{
R. Wuhrer *, K. Moran ** and M. R. Phillips * \\ ${ }_{* *}^{*}$ Microstructural Analysis Unit, University of Technology, Sydney, Broadway, Australia. \\ ** Moran Scientific Pty Ltd, P.O. Box 651, Goulburn, NSW, 2580 Australia.
}

X-ray mapping with Silicon Drift detectors (SDD's) and multi-EDS detector systems has become an invaluable analysis technique because the time to perform an X-ray map is reduced considerably. Live x-ray imaging can now been performed with so much data collected in a matter of minutes. The use of multi-EDS detector systems has made this form of mapping even quicker and has also given users the ability to map minor and trace elements very accurately. How the data is collected and summed with multi-EDS detectors is very critical for accurate quantitative X-ray mapping (QXRM).

There is a great deal of further information that can be obtained from x-ray maps. This includes elemental relationship or scatter diagram creation, elemental ratio mapping, chemical phase mapping (CPM) and quantitative x-ray maps. In obtaining quantitative x-ray maps we are able to easily generate atomic number $(Z)$, absorption $(A)$, fluorescence $(F)$, theoretical back scatter coefficient $(\eta)$ and a quantitative total maps from each pixel in the image. This allows us to generate an image corresponding to each factor (for each element present). These images allow us to predict and verify where we are likely to have problems in our images, and are especially helpful to look at possible interface artefacts.

Figure 1 shows the quantitative elemental x-ray maps produced from a hard facing bonded to a chrome steel. This sample has been prepared by the usual metallographic preparation techniques and investigated on a Jeol 733 microprobe with EDS and WDS detectors. The map was collected with three EDS detectors having a combined detector area of $70 \mathrm{~mm}^{2}$. The beam current for this image was $1 \mathrm{nA}$ with a combined input count rate of 20,000cps.

Figure 2 shows three different BSE images, first from electrons, second from calculated $\eta$ using Tungsten $L$ alpha and third from calculated $\eta$ using Tungsten $M$ alpha. The three BSE images show the affect of the depth distribution of the different signals that make up the images. The BSE image from high energy electrons shows the surface detail and atomic number differences to a depth of about $0.02 \mu \mathrm{m}$. The calculated $\eta$ image (from summing $\mathrm{C}_{\mathrm{i}} \mathrm{Z}_{\mathrm{i}}$ ) using Tungsten $\mathrm{L}$ alpha $(8.396 \mathrm{keV}$ ), shows composition to a depth distribution of about $0.7 \mu \mathrm{m}$. The calculated $\eta$ map using $\mathrm{W} \mathrm{M}$ alpha $(1.777 \mathrm{keV})$, shows composition to a depth distribution of about $1 \mu \mathrm{m}$.

The differences in the images are quite dramatic. The calculated $\eta$ images are also affected by statistical variations, especially with the W L alpha line, as this line is a low intensity line compared with the W M alpha line, but even with this an almost 3D affect (W M alpha line) in the calculated $\eta$ image can be seen when looking around the dendritic growths. The change in spatial resolution is also obvious between the three images.

Figure 3 shows the $\mathrm{Z}, \mathrm{A}$ and $\mathrm{F}$ correction factor maps for Chromium. Because of the elements present in this sample there are very large variations in the Z, A and F correction factors. Chromium 
is a good example to show the fluorescence affect from Iron and atomic number affect from Tungsten. Each element present has its own set of ZAF images.

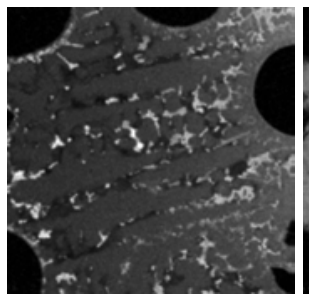

a.

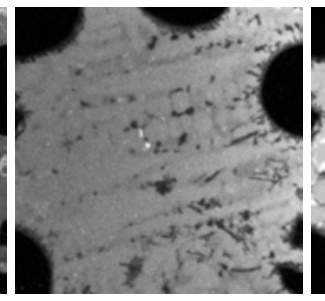

b.

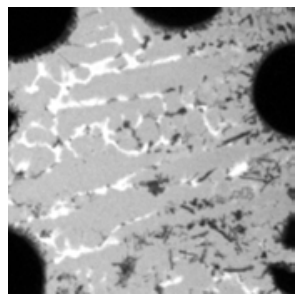

c.

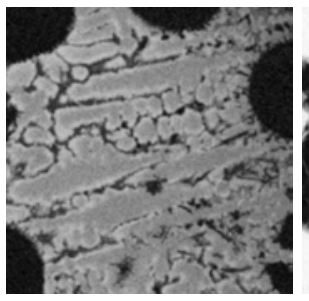

d.

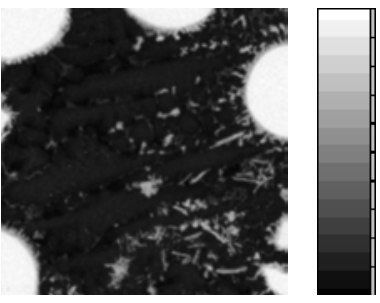

e.

Figure 1: Quantitative (wt\%) elemental x-ray maps produced from a hard facing bonded to a chrome steel. Map collected at $20 \mathrm{keV}, 512 \times 512$ pixels and 12 hours. HWOF $=100 \mu \mathrm{m}$.

a. $\mathrm{Cr} 0-20 \%$, b. Fe $0-30 \%$, c. Ni 0-70\%, d. Cu 0-30\%, e. WC 0-100\%wt.

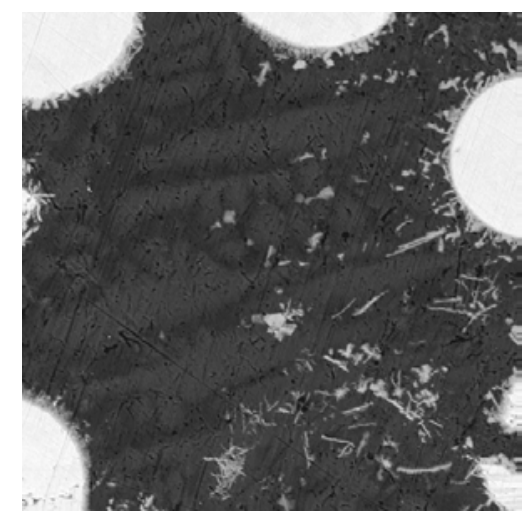

a.

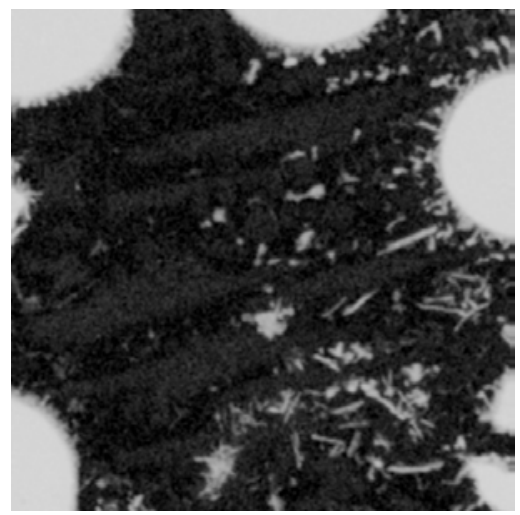

b.

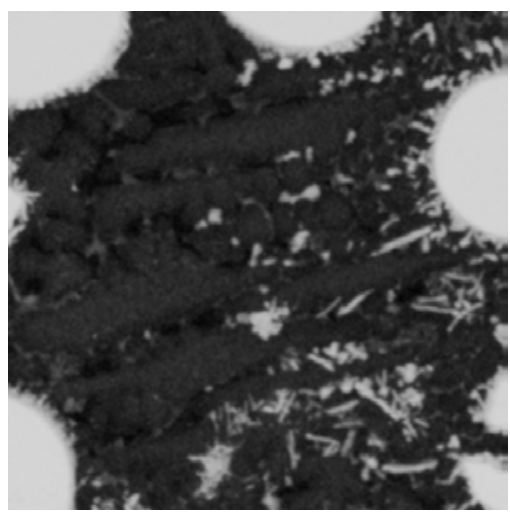

c.

Figure 2: BSE images using a. electrons, b. calculated BSE coefficient ( $\eta$ ) using Tungsten L alpha and c. calculated $\eta$ using Tungsten $\mathrm{M}$ alpha.

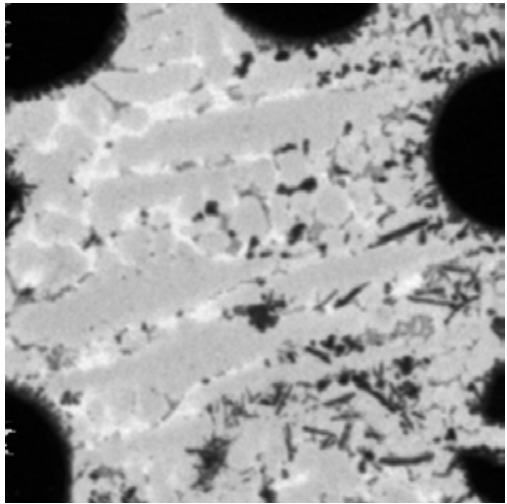

a.

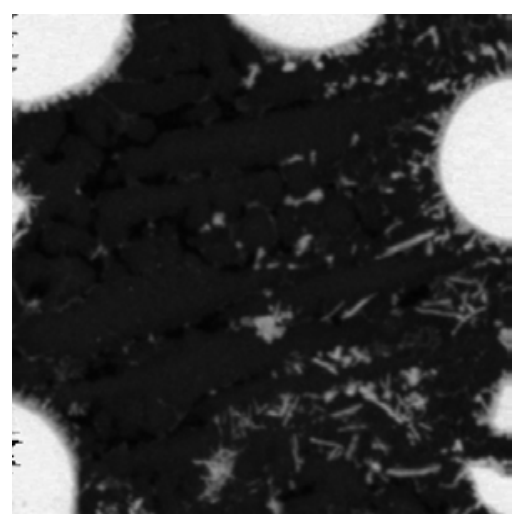

b.

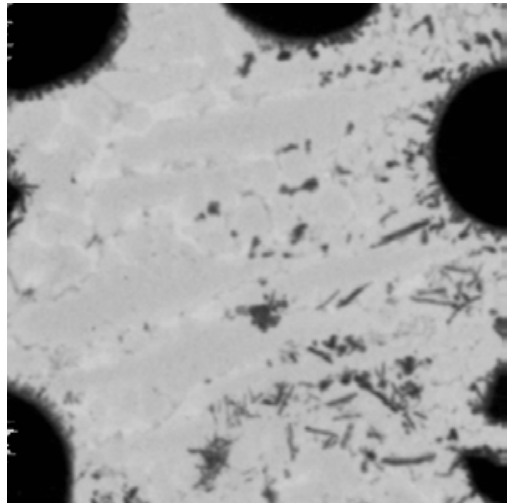

c.

Figure 3: a. Fluorescence factor image for Chromium where the fluorescence factor varies considerably in this case from 1(black) to 1.1(white), b. Atomic number factor image for Chromium where the atomic number factor varies considerably in this case from 1(black) to 1.37(white) and c. Absorption factor image for Chromium where the absorption factor varies in this case from 0.9 (black) to 1 (white). 